\title{
Enhancement of the ERMIN urban dose and remediation model to account for physicochemical properties of contamination
}

\section{T. Charnock}

Centre for Radiation, Chemical and Environmental Hazards, Public Health England, Chilton, Didcot OX11 0RQ, UK.

\begin{abstract}
The European Model for Inhabited Areas (ERMIN) predicts long-term doses and other consequences following contamination of built-up areas by airborne radioactivity. Central to ERMIN are empirical models of the longterm contamination on urban surfaces taking into account weathering and clean-up. Previous versions were largely based on the behaviour of caesium following the Chernobyl accident. Recommendations from a review on the physicochemical properties of particles have allowed enhancement of ERMIN to make it appropriate to a wider range of situations, in particular those where there may be a large proportion of insoluble fuel particles. Weathering experiments in which building materials were contaminated and weathered outdoors have reinforced some of the retention assumptions made by ERMIN but also suggest ways in which future versions of the model might be developed. This paper describes the changes made to ERMIN while maintaining its operational utility within decision support systems and it gives an example of how different assumptions regarding the physicochemical make up of particles can affect results.
\end{abstract}

Keywords: countermeasure simulation / remediation / decision support / surface retention

\section{Introduction}

The European Model for Inhabited Areas (ERMIN) predicts long-term doses and other consequences after contamination of built-up areas by airborne radioactivity. Central to ERMIN are empirical models of the long-term contamination on different urban surfaces that account for weathering and clean-up (Charnock, 2010). Previous versions of ERMIN have largely been based on the behaviour of caesium following the Chernobyl accident. Following a review of literature that focussed on the physicochemical properties of particles, both ERMIN parameters and models have been made appropriate to a wider range of situations, in particular situations with a large proportion of insoluble fuel particles.

Experiments in which different building materials were contaminated with radionuclides and allowed to weather outdoors for a year have reinforced some of the retention assumptions made by ERMIN but also suggest ways in which the model might be further enhanced.

This paper describes the changes made to ERMIN while maintaining its operational utility and it gives an example of how different assumptions regarding the physicochemical make up of particles can affect results. The new version is ERMIN2.1.

\section{Implementation of prepare recommendations}

Under the PREPARE project, a review was conducted to provide recommendations for taking into account various plausible physicochemical forms of contaminants in an incident involving airborne radioactivity (Andersson, 2016). The recommendations are for decision support systems so while the aim is the improvement of models and data this cannot be at the expense of operational requirements. ERMIN is focussed on the recovery phase of an incident and perhaps has less operational constraints than other modules focussed on the fast moving emergency phase, nevertheless it is important that it remains useable. This means that the data requirements placed on users or on the models it links with should not be onerous and run times should remain short.

Previous versions of ERMIN had the capability to use radionuclide specific initial deposition, retention and clean-up parameters, but in reality mostly used a single set of values based on caesium supplemented with limited data for a few other radionuclides. ERMIN2.1 now recognises four particle size groups; "soluble aerosols" and fuel particles with aerodynamic diameter size ranges " $2-5 \mu \mathrm{m}$ ", "5-10 $\mu \mathrm{m}$ " and " $10-20 \mu \mathrm{m}$ " and it is the relative proportions of these for any given radionuclide that are important in defining the radionuclide's long term behaviour. As an example Table 1 gives the retention parameters for roads in the current and previous versions.

ERMIN has now been adapted so that it can link with atmospheric dispersion models (ADM) that provide deposition by particle size, but it is also important for it to remain compatible with models that only provide total depositions, measurements of total deposition or notional values of deposition required for exploratory studies. Rather than forcing the user to add additional particle size information for each radionuclide which would impact on usability, four 
Table 1. Retention parameters for roads ${ }^{\mathrm{a}}$ in ERMIN.

\begin{tabular}{llllll}
\hline ERMIN model version & $\mathrm{A}^{\mathrm{a}}$ & $\mathrm{T} 1$ & $\mathrm{~A} 2$ & $\mathrm{~T} 2$ \\
\hline ERMIN 2 & & 0.7 & 0.4 years & 0.3 & 3 years \\
ERMIN 2.1 & Soluble aerosols & 0.7 & 0.4 years & 0.3 & 3 years \\
& Fuel particles $2-5 \mu \mathrm{m}$ & 1 & 60 days & - & - \\
& Fuel particles 5-10 $\mu \mathrm{m}$ & 1 & 30 days & - & - \\
& Fuel particles $10-20 \mu \mathrm{m}$ & 1 & 30 days & - & -
\end{tabular}

${ }^{a}$ ERMIN uses a double exponential for retention on roads, where A1 and A2 are the fractions of material in the first and second compartments and $\mathrm{T} 1$ and $\mathrm{T} 2$ are the retention half-lives.

Table 2. \% Partitioning of caesium, plutonium and ruthenium into particle size groups.

\begin{tabular}{|c|c|c|c|c|c|c|c|c|c|c|c|c|}
\hline \multirow[t]{2}{*}{ Scenario } & \multicolumn{3}{|c|}{ Soluble aerosols } & \multicolumn{3}{|c|}{ Fuel particles $2-5 \mu \mathrm{m}$} & \multicolumn{3}{|c|}{ Fuel particles $5-10 \mu \mathrm{m}$} & \multicolumn{3}{|c|}{ Fuel particles $10-20 \mu \mathrm{m}$} \\
\hline & Cs & $\mathrm{Pu}$ & $\mathrm{Ru}$ & Cs & $\mathrm{Pu}$ & $\mathrm{Ru}$ & Cs & $\mathrm{Pu}$ & $\mathrm{Ru}$ & Cs & $\mathrm{Pu}$ & $\mathrm{Ru}$ \\
\hline 1 & 100 & - & - & 0 & - & - & 0 & - & - & 0 & - & - \\
\hline 2 & 50 & 0 & 0 & 2.5 & 5 & 5 & 22.5 & 45 & 45 & 25 & 50 & 50 \\
\hline 3 & 100 & - & 95 & 0 & - & 0.25 & 0 & - & 2.25 & 0 & - & 2.5 \\
\hline 4 & 50 & 0 & 50 & 2.5 & 5 & 2.5 & 22.5 & 45 & 22.5 & 25 & 50 & 25 \\
\hline
\end{tabular}

$\mathrm{Pu}$ is not expected except as part of a fuel particle and $\mathrm{Ru}$ is not expected except as a fuel particle or with oxidation.

broad scenarios have been developed from which the user may select. The scenarios are:

- No fuel particles and no oxidation. In this scenario no fuel particles have been released or the area of deposition is sufficiently distant from the point of release that few fuel particles which have relatively large deposition velocities have been transported so far.

- Significant fuel particles and no oxidation. In this scenario significant fuel particles have been released and the area of deposition is sufficiently close that the high deposition velocity of the fuel particles means they are deposited in relatively large amounts.

- No fuel particles with significant oxidation. This is the same as scenario 1 however there was sufficient oxygen available during particle formation for oxidation to occur.

- Significant fuel particles with significant oxidation. This is the same as scenario 2 but with oxidation during particle formation, as with scenario 3 .

Oxidation is only significant for ruthenium, which can be oxidised to a volatile form; without oxidation its high melting point would prevent it being depleted from the fuel material. As an example Table 2 shows the partitioning of caesium, plutonium and ruthenium that ERMIN uses in each scenario.

\section{Surface retention experiments}

The surface retention experiments performed under the PREPARE project (Brown et al., 2016) correspond to the soluble aerosols group. Undertaking experiments in real weather conditions has meant that interpretation of the results has not been straightforward. However, there are differences between radionuclides, which suggest that there may be a need for subcategories of this particle group in the future. Weathering data for surface materials not yet modelled in ERMIN, such as wood and glass, will also prove useful if new environments such as a glass fronted office block are added to ERMIN. Another modification suggested by the experimental results is that an initial reduction in contamination at the "time of first rain" might better represent weathering from some surfaces; this could be added and would not be too onerous for users to provide. Other factors that may influence weathering in specific weather conditions are discussed in Brown et al. (2016).

\section{Example results}

One of the most useful outputs from ERMIN is the contribution of different urban surfaces to total dose, as this will allow decision makers to target a surface for clean-up. Figure 1 shows the difference that scenario choice makes for a deposition of Caesium-137.

\section{Conclusion}

ERMIN has been enhanced to account for physicochemical properties of particles, in particular the presence or 

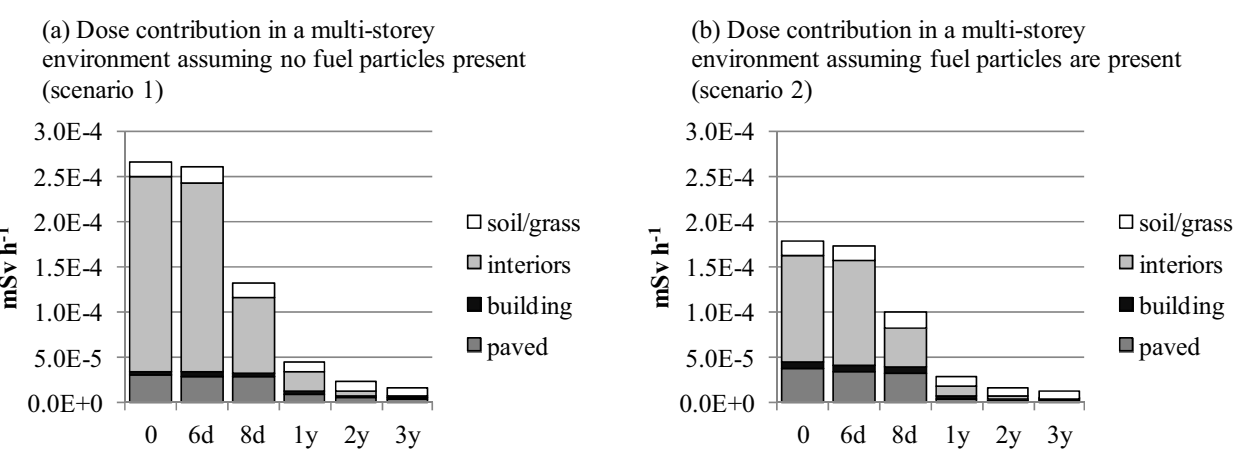

Figure 1. Example ERMIN output showing predicted dose rates in a multi-storey building environment assuming $90 \%$ time spent indoors, a deposition of Cs-137 and washing of interior surfaces at one week. In this example, if a proportion of the contamination is in the form of fuel particles as in the case for graph (b), then interior deposition is less.

absence of insoluble fuel particles. It can now be run with an ADM that provides particle size information for each radionuclide and alternatively four scenarios have been developed to set consistent particle group partitioning when only total deposition is available from an ADM or from measurements.

Acknowledgement. The research leading to these results has received funding from the European Atomic Energy Community Seventh Framework Programme FP7/2012-2013 under grant agreement 323287.

\section{References}

Andersson K. (2016) Physico-chemical properties of radionuclides emitted as particulate matter, Radioprotection, 51 (HS2), S97-S99.

Brown J., Ewers L.W., Youngman M.J. (2016) An experimental study on natural weathering of radionuclides from urban surfaces for aerosols deposited in wet and dry conditions, Radioprotection, $\mathbf{5 1}$ (HS2), S109-S112.

Charnock T.W. (2010) The European model for inhabited areas (ERMIN) - developing a description of the urban environment, Radioprotection 45 (5), S55-S61.

Cite this article as: T. Charnock. Enhancement of the ERMIN urban dose and remediation model to account for physicochemical properties of contamination. Radioprotection 51(HS2), S105-S107 (2016). 